PROCEEDINGS OF THE

AMERICAN MATHEMATICAL SOCIETY

Volume 120, Number 4, April 1994

\title{
A NECESSARY CONDITION FOR AN ELLIPTIC ELEMENT TO BELONG TO A UNIFORM TREE LATTICE
}

\author{
YING-SHENG LIU
}

(Communicated by Ronald M. Solomon)

\begin{abstract}
Let $X$ be a universal cover of a finite connected graph, $G=$ $\operatorname{Aut}(X)$, and $\Gamma$ a group acting discretely and cocompactly on $X$, i.e., a uniform lattice on $X$. We give a necessary condition for an elliptic element of $G$ to belong to a uniform lattice or to the commensurability group. By using this condition, we construct some explicit examples.
\end{abstract}

Continuing the classic Bass-Serre theory on graphs of groups [S], Bass developed the covering theory for graphs of groups [B]. Using this, Bass and Kulkarni developed the uniform tree lattices theory in their joint paper [BK]. In that paper they obtained a lot of important results. It is fruitful to think of $(G, X, \Gamma)$ as a combinatorical analogue of $\left(\mathrm{PSL}_{2}(\mathbf{R})\right.$, upper half plane, fuchsian group).

Let $X$ be a 'uniform tree', i.e., the universal cover of a finite connected graph, $G=\operatorname{Aut}(X)$, equipped with compact open topology. The subgroup $H<G$ is discrete iff every vertex stabilizer $H_{x}$ for $x \in V X$ is finite, where $V X$ is the set of all vertices of $X$. We call $\Gamma<G$ a uniform $X$-lattice if $\Gamma$ is discrete and the quotient graph $\Gamma \backslash X$ is finite (i.e., $V X$ has only finitely many $\Gamma$-orbits). Let $\Gamma_{0}, \Gamma_{1}$ be subgroups of $G . \Gamma_{0}$ and $\Gamma_{1}$ are said to be commensurable (denoted $\left.\Gamma_{0} \sim \Gamma_{1}\right)$, if the index $\left[\Gamma_{i}: \Gamma_{0} \cap \Gamma_{1}\right]$ is finite for $i=0,1$. The commensurator (or "virtual normalizer') of $\Gamma$ in $G$ is the group $C_{G}(\Gamma)=\left\{g \in G \mid g \Gamma g^{-1} \sim \Gamma\right\}$. It was shown in [BK] that, up to $G$-conjugacy, any two uniform lattices in $G$ are commensurable. Thus the commensurator $C_{G}(\Gamma)$ of a uniform lattice $\Gamma \leq G$ is, up to conjugacy, independent of $\Gamma$; we denote it by $C(X)$. It is proved in [L1] that $C(X)$ is dense in $G$, which was conjectured in [BK].

In this paper, we give a necessary condition for an elliptic element (i.e., one having fixed points) of $G$ to belong to a uniform lattice or to the commensurability group $C(X)$. By this condition, it is then easy to construct some automorphisms of $X$ which do not belong to a uniform lattice, nor do they belong to $C(X)$.

We address here the following questions:

Question. Let $X$ be a uniform tree, $G=\operatorname{Aut}(X), g \in G$. When is there a uniform $X$-lattice $\Gamma$ : (a) such that $g \in \Gamma$ ?; (b) such that $g \in C_{G}(\Gamma)$ ?

Received by the editors July 19, 1992.

1991 Mathematics Subject Classification. Primary 05C25, 05 C05.

(C) 1994 American Mathematical Society $0002-9939 / 94 \$ 1.00+\$ .25$ per page 
We begin by quoting

Proposition 1 [BK, (4.2) Conjugacy Theorem]. If $g$ is hyperbolic (i.e., acting on $X$ freely), then $g$ belongs to a uniform lattice.

So the case of main interest is when $g$ is of finite order. The following notion, due to Gelfand, will be useful for our discussion.

Definition. Let $G$ be a locally compact group. An element $u \in G$ will be called $G$-unipotent if the closure of its $G$-conjugacy class $C_{G}(u)$ contains 1 , where $C_{G}(u)=\left\{g u g^{-1} \mid g \in G\right\}$.

Lemma 1. Assume that $\Gamma \backslash G$ is compact, in the sense that $G=K \cdot \Gamma$ for some compact set $K \subset G$. If $\sigma \in G$ is $G$-unipotent then the closure of its $\Gamma$-conjugacy class $C_{\Gamma}(\sigma)$ contains 1 , where $C_{\Gamma}(\sigma)=\left\{\gamma \sigma \gamma^{-1} \mid \gamma \in \Gamma\right\}$.

Proof. Say

$$
1=\lim _{n} g_{n} \sigma g_{n}^{-1}, \quad g_{n} \in G, n=1,2, \ldots
$$

Write $g_{n}=k_{n} \gamma_{n}$, where $k_{n} \in K, \gamma_{n} \in \Gamma$.

Passing to a subsequence we can (compactness of $K$ ) assume that $k_{n} \rightarrow k$ for some $k \in K$. Then

$$
1=\lim _{n} k_{n} \gamma_{n} \sigma \gamma_{n}^{-1} k_{n}^{-1}=\lim _{n} k \gamma_{n} \sigma \gamma_{n}^{-1} k^{-1} \text {, }
$$

and so

$$
1=\lim _{n} \gamma_{n} \sigma \gamma_{n}^{-1}, \quad \gamma_{n} \in \Gamma \text {. Q.E.D }
$$

Proposition 2. Let $\Gamma \in \operatorname{Lat}_{u}(X)$; then $C_{G}(\Gamma)$, in particular $\Gamma$, contains no $G$-unipotent element $\neq 1$.

Proof. Suppose that $\sigma \in C_{G}(\Gamma)$ is $G$-unipotent.

Put $\Gamma^{\prime}=\Gamma \cap \sigma \Gamma \sigma^{-1}$, a subgroup of finite index in $\Gamma$. Applying Lemma 1 to $\Gamma^{\prime}$, we have $1=\lim _{n} \gamma_{n} \sigma \gamma_{n}^{-1}$ with $\gamma_{n} \in \Gamma^{\prime}$. Hence,

$$
\sigma^{-1}=\lim _{n} \sigma^{-1} \gamma_{n} \sigma \gamma_{n}^{-1} \text {. }
$$

But, for each $n,\left(\sigma^{-1} \gamma_{n} \sigma\right) \gamma_{n}^{-1} \in\left\langle\sigma^{-1} \Gamma^{\prime} \sigma, \Gamma^{\prime}\right\rangle \leq \Gamma$, and $\Gamma$ is discrete. Hence, for $n \gg 0$,

$$
\sigma^{-1}=\sigma^{-1} \gamma_{n} \sigma \gamma_{n}^{-1}
$$

whence, $\gamma_{n} \sigma \gamma_{n}^{-1}=1$, i.e., $\sigma=1$. Q.E.D.

Thus we get a necessary condition for an elliptic $g \neq 1$ to belong to a uniform tree lattice or $C(X)$ that $g$ is not a $G$-unipotent element.

Lemma 2. An element $\sigma \in G$ is G-unipotent iff it is elliptic and its tree of fixed points contains a $G$-translate of any given finite subtree.

Proof. Assume that $\sigma \in G$ is $G$-unipotent. Thus, by the definition, there is a sequence $\left\{g_{n} \in G, n=1,2, \ldots\right\}$ such that $\lim _{n} g_{n}^{-1} \sigma g_{n}=1$. In other words, for any given finite subtree $Y$ of $X$ and for $n \gg 0$, we have $g_{n}^{-1} \sigma g_{n}|Y=\mathrm{id}| Y$, i.e., $\sigma\left|\left(g_{n} Y\right)=\mathrm{id}\right|\left(g_{n} Y\right)$. So, $\sigma$ is elliptic and its tree of fixed points contains $g_{n} Y$, where $g_{n} \in G$ and $Y$ is any given finite subtree of $X$.

Conversely, suppose that $\sigma$ is elliptic and its tree of fixed points contains a $G$-translate of any given finite subtree. 
For $a \in V X$, put $B_{a}(n)=\{x \in V X \mid d(a, x) \leq n\}$. Then $\left\{B_{a}(n), n=\right.$ $1,2, \ldots\}$ is a sequence of finite subtrees of $X$. For each $B_{a}(n)$, by the assumption, there is $g_{n} \in G$, such that

$$
\sigma\left|\left(g_{n} B_{a}(n)\right)=\mathrm{id}\right|\left(g_{n} B_{a}(n)\right),
$$

i.e.,

$$
g_{n}^{-1} \sigma g_{n}\left|B_{a}(n)=\mathrm{id}\right| B_{a}(n), \quad g_{n} \in G, n=1,2, \ldots
$$

So, $\lim _{n} g_{n}^{-1} \sigma g_{n}=1, g_{n} \in G$. Q.E.D.

Now, it is easy to construct $G$-unipotent elements of finite order, which thus lie in no uniform lattice (or even the commensurator of one).

Example 1. Let $X$ be the following virtually linear tree:

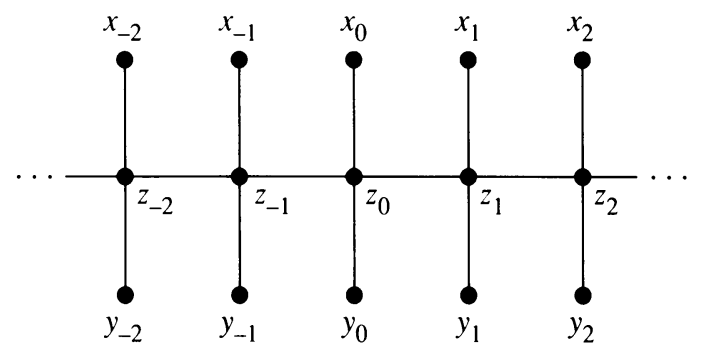

Clearly, $X$ is a uniform tree. In fact, let $g \in \operatorname{Aut}(X)$ be defined by

$$
g\left(x_{n}\right)=x_{n+1}, \quad g\left(y_{n}\right)=y_{n+1}, \quad g\left(z_{n}\right)=z_{n+1}, \quad n=0, \pm 1, \pm 2, \ldots,
$$

then $\langle g\rangle$ is a uniform lattice of $X:\langle g\rangle$ is discrete and $\langle g\rangle \backslash X$ is finite.

Define $\sigma \in G=\operatorname{Aut}(X)$, such that $\sigma\left(x_{0}\right)=y_{0}, \sigma\left(y_{0}\right)=x_{0}$, and $\sigma$ acts on $X-\left\{x_{0}, y_{0}\right\}$ trivially.

Clearly, the subtree of fixed points of $\sigma$ contains a $G$-translate of any given finite subtree of $X$. By Lemma $2, \sigma$ is a nontrivial $G$-unipotent. Hence, by Proposition 2, $\sigma$ does not belong to any uniform lattice nor even to the commensurator of any uniform lattice.

Example 2. Let $X$ be the Cayley tree $\operatorname{Cay}(F(x, y),\{x, y\})$, where $F(x, y)$ is a free group on a basis $\{x, y\}$. Let $\alpha \in \operatorname{Aut}(F(x, y))$, such that $\alpha(x)=y$, $\alpha(y)=x$. Put

$$
P=\{u \in F(x, y) \mid \text { reduced word of } u \text { begins with } x \text { or } y\} .
$$

Note that $\alpha$ defines an automorphism of $X$ and $\alpha P=P$. Define $\sigma \in$ $\operatorname{Aut}(X)$ by

$$
\sigma(u)= \begin{cases}\alpha(u) & \text { if } u \in P \\ u & \text { if } u \notin P .\end{cases}
$$


$X=\operatorname{Cay}(F(x, y), x, y):$

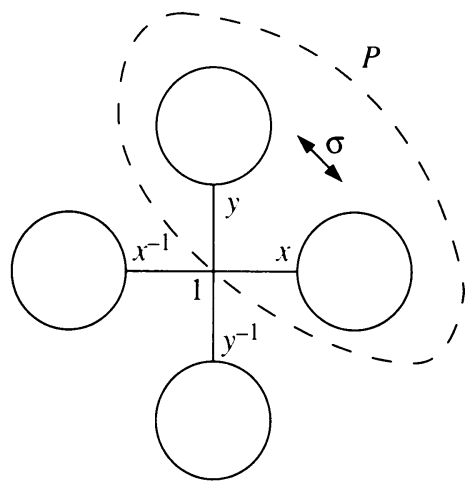

Since $\sigma$ switches two branches of $X$ and fixes the other two branches, the subtree of fixed points of $\sigma$ contains $G$-translate of any given finite subtree of $X$. By Lemma 2, $\sigma$ is a nontrivial $G$-unipotent. Hence, $\sigma$ lies in no uniform lattice nor even the commensurator of one.

On the other hand, we have

Proposition 3. Let $\Gamma \leq G$ be a uniform lattice and $F \leq C_{G}(\Gamma)$ a subgroup such that $F \cdot \Gamma / \Gamma$ is finite. Then $F \leq \Gamma^{\prime}$ for some $\Gamma^{\prime} \sim \Gamma$.

Proof. We may assume that $F \cdot \Gamma=S \cdot \Gamma$, where $S$ is a finite subset of $C_{G}(\Gamma)$. Put

$$
\Gamma_{0}=\bigcap_{g \in F \cdot \Gamma} g \Gamma g^{-1}=\bigcap_{s \in S} s \Gamma s^{-1} .
$$

As $s \in C_{G}(\Gamma), s \Gamma s^{-1} \sim \Gamma$ for each $s \in S$. Since the intersection of two subgroups of finite index has finite index, it follows that a commensurability class of subgroups of $G$ is stable under finite intersection. Thus the finite intersection $\Gamma_{0}$ is commensurable with $\Gamma$. And, clearly, $\Gamma_{0}$ is normalized by $F$, i.e., $F \leq N_{G}\left(\Gamma_{0}\right)$. According to [BK, Corollary (6.4)], $\Gamma_{0} \backslash N_{G}\left(\Gamma_{0}\right)$ is finite, so $N_{G}\left(\Gamma_{0}\right) \sim \Gamma_{0} \sim \Gamma$. Thus the proposition is proved by taking $\Gamma^{\prime}=N_{G}\left(\Gamma_{0}\right)$.

Remark. Proposition 3 applies notably when $F \leq C_{G}(\Gamma)$ is finite or when $F=$ $\langle g\rangle$ with $g^{n} \in \Gamma$ for some $n>0$.

\section{ACKNOWLEDGMENT}

The above results formed a portion of my doctoral dissertation [L2]. I am very grateful to my advisor Hyman Bass for all of his help and encouragement. He has always been concerned about my work and generous with his time and ideas.

I also thank Ravi Kulkarni, John Morgan, and Guoliang Yu for their interest and helpful comments. At last, I thank the referee for his careful reading and good suggestions.

\section{REFERENCES}

[AB] R. Alperin and H. Bass, Length functions of group actions on $\Lambda$-trees, Combinatorial Group Theory and Topology, Ann. of Math. Stud., no. 111, Princeton Univ. Press, Princeton, NJ, 1987 , pp. $265-378$. 
[B1] H. Bass, Covering theory for graphs of groups, J. Pure Appl. Algebra (to appear).

[B2] - Group actions on non-archimedean trees, Aboreal Group Theory (Roger C. Alperin, ed.), Math. Sci. Res. Inst. Publ., vol. 19, Springer-Verlag, New York, 1988, pp. 69-131.

[BK] H. Bass and R. Kulkarni, Uniform tree lattices, J. Amer. Math. Soc. 3 (1990), 843-902.

[K] R. Kulkarni, Lattices on trees, automorphism of graphs, free groups, surfaces, preprint, CUNY, September 1988.

[KPS] A. Karass, A. Pietrowski, and D. Solitar, Finite and infinite cyclic extensions of free groups, Australian Math. Soc. 16 (1973), 458-466.

[L1] Y. Liu, Density of commensurability groups of uniform tree lattices, J. Algebra (to appear).

[L2] _ Commensurability groups of uniform tree lattices, Columbia Univ. Dissertation, 1991.

[Lub] A. Lubotzky, Trees and discrete subgroups of Lie groups over local fields, Bull. Amer. Math. Soc. (N.S.) 20 (1988), 27-31.

[SP] J.-P. Serre, Trees, Springer-Verlag, New York, 1980.

Department of Mathematics, State University of New York, College at Plattsburgh, Plattsburgh, New York 12901

E-mail address: liuys@snyplava.bitnet 